\title{
REPRESENTASE QUATERNION DENGAN MENGGUNAKAN NOTASI $\mathbb{C}_{j}-$ pair
}

Muh. Irwan ${ }^{\mathrm{i}}$

Erniwati Jalil ${ }^{\text {ii }}$

${ }^{i}$ Prodi Matematika FST, UINAM, muhirwan @uin-alauddin.ac.id

ii Prodi Matematika, Fakultas MIPA, UMMA, erniwatijalil@gmail.com

ABSTRAK,Quaternion merupakan perluasan bilangan kompleks dalam empat dimensi (4D). dalam melakukan representasi bilangan quaternion terdapat beberapa cara yang bisa digunakan, seperti bentuk polar (Polar Form),The Cayley-Dickson, The ortho-split dan notasi $\mathbb{C}_{j}-$ Pair. Pada artikel ini digunakan notasi $\mathbb{C}_{j}-$ Pairuntuk merepresentasikan quaternion serta memperlihatkan kasus khusus $\mathbb{C}_{j}-$ Pair dalam aplikasinya pada perkalian bikompleks quaternion.

Kata Kunci:Quaternion, Representasi Quaternion, $\mathbb{C}_{j}-$ Pair, Bicomplex Product Quaternion

\section{PENDAHULUAN}

Bilangan quaternion merupakan pengembangan bilangan kompleks. Yang menarik untuk diperhatikan bahwa pada bilangan quaternion tidak berlaku sifat komutatif terhadap operasi perkalian. Bilangan quaternion pertama kali diperkenalkan oleh Sir Rowman Hamilton padatahun 1805-1865. [5][6][7].

Representasi bilangan quaternion menjadi suatu yang unik untuk dikaji. Beberapa cara yang bisa digunakan untuk melakukan representasi quaternion diataranya, dekomposisi quaternion, bentuk polar quaternion, $C D$ form, CarleyDickson, Ortho-Splitdan $\mathbb{C}_{j}-$ Pair.

Pada artikel ini, akan dibahas tentang $\mathbb{C}_{j}-$ Pair hal ini karena pada notasi $\mathbb{C}_{j}-$ Pair berlaku sifat komutatif perkalian. Sedangkan pada bilangan quaternion sendiri tidak berlaku sifat komutatif perkalian. Adanya kontrakdiksi tersebut, sehingga pada artikel ini akan diperlihatkan beberapa teknik modifikasi secara aljabar sehingga kondisi perkalian dua bilangan quaternion dapat memenuhi sifat komutatif.

\section{TINJAUANPUSTAKA}

\section{Bilangan Quaternion}

Himpunan bilangan quaternion dituliskan dengan simbol $\mathbb{H}$ sebagai penghargaan bagi Sir William Roman Hamilton, dimana elemen- elemen dari bilangan quaternion merupakan kombinasi linear dari bilangan skalar riil dan tiga bagian imajiner $\boldsymbol{i}, \boldsymbol{j}$ dan $\boldsymbol{k}$ yang dituliskan sebagai $[1,2,3]$,

$\mathbb{H}=\left\{q=q_{0}+\boldsymbol{i} q_{1}+\boldsymbol{j} q_{2}+\boldsymbol{k} q_{3} \mid q_{0}, q_{1}, q_{2}, q_{3} \in\right.$ $\mathbb{R}\}$.

Jika $q_{2}$ dan $q_{3}$ bernilai nol maka persamaan (1) merupakan suatu bilangan kompleks. Sedangkan jika $\quad q_{0}=q_{1}=q_{2}=q_{3}=0$, maka persamaan(1) merupakan elemen identitas penjumlahan quaternion. Jika $q_{0}=1, q_{1}=q_{2}=$ $q_{3}=0$ maka persamaan(1) disebut elemen identitas perkalian quaternion (J.P. Morais, dkk. 2012). Selanjutnya, persamaan (1) dapat dituliskan menjadi

$$
q=S c(q)+\boldsymbol{q}
$$

Dalam hal $\operatorname{iniSc}(q)=q_{0}$ adalah bagian skalar dari $q$, dan $\boldsymbol{q}=\boldsymbol{i} q_{1}+\boldsymbol{j} q_{2}+\boldsymbol{k} q_{3}$ sebagai bagian vektor (vector part) dari $q$ [6]. Perkalian elemenelemen dari suatu bilangan quaternion berdasarkan aturan Hamilton dapat dituliskan sebagai

$$
\begin{aligned}
& \boldsymbol{i}^{2}=\boldsymbol{j}^{2}=\boldsymbol{k}^{2}=\boldsymbol{i} \boldsymbol{j} \boldsymbol{k}=-1 . \\
& \boldsymbol{j} \boldsymbol{k}=-\boldsymbol{k} \boldsymbol{j}=\boldsymbol{i}, \boldsymbol{i} \boldsymbol{j}=-\boldsymbol{j} \boldsymbol{i}=\boldsymbol{k} \\
& \boldsymbol{j} \boldsymbol{k}=-\boldsymbol{k} \boldsymbol{j}=\boldsymbol{i} .
\end{aligned}
$$

Dapat diperhatikan bahwa operasi penjumlahan dan pengurangan quaternion dilakukan seperti pada operasi penjumlahan dan pengurangan suku banyak. Jikap, $q \in \mathbb{H}$ dengan $p=p_{0}+\boldsymbol{i} p_{1}+$ $\boldsymbol{j} p_{2}+\boldsymbol{k} p_{3} \quad$ dan $\quad q=q_{0}+\boldsymbol{i} q_{1}+\boldsymbol{j} q_{2}+\boldsymbol{k} q_{3}$ dimana $p_{0}, p_{1}, p_{2}, p_{3}, q_{0}, q_{1}, q_{2}, q_{3} \in \mathbb{R}$ maka $p+q=\left(p_{0}+q_{0}\right)+\boldsymbol{i}\left(p_{1}+q_{1}\right)+\boldsymbol{j}\left(p_{2}+\right.$ $\left.q_{2}\right)+\boldsymbol{k}\left(p_{3}+q_{3}\right)$.

Dengan cara yang sama, operasi pengurangan pada bilangan quaternion

$p-q=\left(p_{0}-q_{0}\right)+\boldsymbol{i}\left(p_{1}-q_{1}\right)+\boldsymbol{j}\left(p_{2}-\right.$ $\left.q_{2}\right)+\boldsymbol{k}\left(p_{3}-q_{3}\right)$.

Selanjutnya,operasi perkalian $p$ dan $q$ dilakukan berdasarkan perkalian polinom yaitu 
$p q$

$=\left(p_{0}+\boldsymbol{i} p_{1}+\boldsymbol{j} p_{2}+\boldsymbol{k} p_{3}\right)\left(q_{0}+\boldsymbol{i} q_{1}+\boldsymbol{j} q_{2}\right.$

$\left.+\boldsymbol{k} q_{3}\right)$

$=p_{0} q_{0}-\left(p_{1} q_{1}+p_{2} q_{2}+p_{3} q_{3}\right)$

$+p_{0}\left(\boldsymbol{i} q_{1}+\boldsymbol{j} q_{2}+\boldsymbol{k} q_{3}\right)$

$+q_{0}\left(\boldsymbol{i} p_{1}+\boldsymbol{j} p_{2}+\boldsymbol{k} p_{3}\right)+\boldsymbol{i}\left(p_{2} q_{3}-p_{3} q_{2}\right)$

$+\boldsymbol{j}\left(p_{1} q_{3}-p_{3} q_{1}\right)+\boldsymbol{k}\left(p_{1} q_{2}-p_{2} q_{1}\right)$

$=p_{0} q_{0}-\boldsymbol{p} . \boldsymbol{q}+p_{0} \boldsymbol{q}+q_{0} \boldsymbol{p}+\boldsymbol{p} \times \boldsymbol{q}$

dimana $\boldsymbol{p} \cdot \boldsymbol{q}=\left(p_{1} q_{1}+p_{2} q_{2}+p_{3} q_{3}\right)$ adalah hasil kali titik (dot product) dan $\boldsymbol{p} \times \boldsymbol{q}=$ $\boldsymbol{i}\left(p_{2} q_{3}-p_{3} q_{2}\right)+\boldsymbol{j}\left(p_{1} q_{3}-p_{3} q_{1}\right)+\boldsymbol{k}\left(p_{1} q_{2}-\right.$

$\left.p_{2} q_{1}\right)$. Hasil perkalian $p q$ dengan $q p$ tidak selalu sama, ini karena perkalian silang dari $\boldsymbol{p}$ dan $\boldsymbol{q}$ tidak komutatif. Sehingga dapat dikatakan bahwa $p q=q p$ jika dan hanya jika $p_{0}=q_{0}$ dan $\boldsymbol{p}=\boldsymbol{q}$.

Definisi 2.1.Untuk sebarang $p \in \mathbb{H}$ dengan $p=$ $p_{0}+\boldsymbol{i} p_{1}+\boldsymbol{j} p_{2}+\boldsymbol{k} p_{3}$ dimana $p_{0}, p_{1}, p_{2}, p_{3} \in \mathbb{R}$, konjugasi dari $p$ adalah

$$
\begin{aligned}
& \bar{p}=\overline{p_{0}+\boldsymbol{i} p_{1}+\boldsymbol{j} p_{2}+\boldsymbol{k} p_{3}} \\
& =p_{0}-\boldsymbol{i} p_{1}-\boldsymbol{j} p_{2}-\boldsymbol{k} p_{3} .
\end{aligned}
$$

Berdasarkan persamaan (7) dapat dipahami bahwa konjugasi dari $p$ merupakan perubahan tanda pada bagian imajiner sedangkan bagian riil tidak berubah tanda. Hasil perkalian konjugasi quaternion adalah suatu anti-involusi, hal ini dapat ditunjukkan bahwa

$\overline{p q}=\bar{q} \bar{p}$ )

yaitu

$\bar{q} \bar{p}$

$=\left(q_{0}-\boldsymbol{i} q_{1}-\boldsymbol{j} q_{2}-\boldsymbol{k} q_{3}\right)\left(p_{0}-\boldsymbol{i} p_{1}-\boldsymbol{j} p_{2}\right.$

$\left.-\boldsymbol{k} p_{3}\right)$

$=q_{0}\left(p_{0}-\boldsymbol{i} p_{1}-\boldsymbol{j} p_{2}-\boldsymbol{k} p_{3}\right)$

$-\boldsymbol{i} q_{1}\left(p_{0}-\boldsymbol{i} p_{1}-\boldsymbol{j} p_{2}-\boldsymbol{k} p_{3}\right)$

$-\boldsymbol{j} q_{2}\left(p_{0}-\boldsymbol{i} p_{1}-\boldsymbol{j} p_{2}-\boldsymbol{k} p_{3}\right)$

$-\boldsymbol{k} q_{3}\left(p_{0}-\boldsymbol{i} p_{1}-\boldsymbol{j} p_{2}-\boldsymbol{k} p_{3}\right)$

$=p_{0} q_{0}-\boldsymbol{p} \cdot \boldsymbol{q}-q_{0} \boldsymbol{p}-p_{0} \boldsymbol{q}-\boldsymbol{p} \times \boldsymbol{q}$.

Dan modulo $p$ dituliskan dengan $|\mathrm{p}|$ dan didefinisikan sebagai

$$
|p|=\sqrt{p_{0}^{2}+p_{1}^{2}+p_{2}^{2}+p_{3}^{2}}
$$

\section{Dekomposisi quaternion}

Misalkan $q=q_{0}+\boldsymbol{i} q_{1}+\boldsymbol{j} q_{2}+\boldsymbol{k} q_{3}$, maka $q$ dapat didekomposisi menjadi

$q=q_{+}+q_{-}=\frac{1}{2}(q+\boldsymbol{i} q \boldsymbol{j})+\frac{1}{2}(q-\boldsymbol{i} q \boldsymbol{j}), \quad$ dari dekomposisi ini sehingga

$$
\left.q_{ \pm}=\left\{q_{0} \pm q_{3}+\boldsymbol{i}\left(q_{1} \mp q_{2}\right)\right\} \frac{1 \pm \boldsymbol{k}}{2}\right) .
$$

\section{METODOLOGI}

Penulisan artikel ini dilakukan dengan langkahlangkah sebagai berikut,

a. Merumuskan notasi $\mathbb{C}_{j}-$ Pair

b. Menguraikan operasi aritmetika quaternion dengan menggunakan notasi $\mathbb{C}_{j}-$ Pair

c. Menguraikan aturan perkalian bikompleks quaternion

d. Merumuskan beberapa sifar perkalian bicompleks quaternion

\section{PEMBAHASAN}

Pada bagian ini, akan dijelaskan lebih jauh mengenai operasi dasar bilangan quaternion.

Diberikan quaternion

$$
q=q_{0}+\boldsymbol{i} q_{1}+\boldsymbol{j} q_{2}+\boldsymbol{k} q_{3} \in \mathbb{H}
$$

\section{Definisi 1.}

Misalkan $q$ dituliskan dalam bentuk $q=u+\boldsymbol{i} v$, dimana $u=q_{0}+\boldsymbol{j} q_{2}$ dan $v=q_{1}+\boldsymbol{j} q_{3} \in \mathbb{C}_{\boldsymbol{j}}$. Quaternion $q$ dipahami sebagai bentuk pasangan bilangan kompleks dengan ordinat $\boldsymbol{j}$. Dan bentuk ini disebut sebagai $\mathbb{C}_{j}-$ Pair. Berikut ini diperlihatkan bahwa notasi $\mathbb{C}_{j}-$ Pair merupakan bentuk lain dari quaternion $q$ [7]. Dengan memperhatikan bahwa,

$$
q=u+i v
$$

Dengan mensubstitusikan nilai $u$ dan $v$. Maka quaternion $q$ menjadi

$$
\begin{aligned}
q & =q_{0}+\boldsymbol{j} q_{2}+\boldsymbol{i}\left(q_{1}+\boldsymbol{j} q_{3}\right) \\
& =q_{0}+\boldsymbol{j} q_{2}+\boldsymbol{i} q_{1}+\boldsymbol{i} \boldsymbol{j} q_{3} \\
& =q_{0}+\boldsymbol{j} q_{2}+\boldsymbol{i} q_{1}+\boldsymbol{k} q_{3} .
\end{aligned}
$$

Quaternion $q$ dapat disebut sebagai pasangan terurut dari $u$ dan $v$ atau dituliskan dengan $(u, v)$. Pada bilangan kompleks $u$ dan $v$ berturut-turut disebut sebagai bagian real dan bagian imajiner. Tentunya untuk quaternion, quaternion $q$ dapat juga disebut sebagai representasi bialngan kompleks. Namun perlu sangat hati-hati untuk mengatakan hal tersebut karena pada hakikatnya $u$ mengandung $q_{0}$ dan $q_{2}$ sedangkan $v$ mengandung $q_{1}$ dan $q_{3}$. Untuk lebih menyederhanakan penulisan quaternion, selanjutnya akan digunakan $q$ sebagai representasi $(u, v)$. 
Lebih jauh, akan dibahas notasi $\mathbb{C}_{j}-$ Pair hubungannya dengan aljabar quaterion.

\section{Penjumlahan}

\section{Teorema 1}

Misalkan $q=(u, v), p=(w, x) \in \mathbb{C}_{j}$ dengan $u=q_{0}+\boldsymbol{j} q_{2}, v=q_{1}+\boldsymbol{j} q_{3}, w=p_{0}+\boldsymbol{j} p_{2}, x=$ $p_{1}+\boldsymbol{j} p_{3}$.

Maka

Bukti

$$
q+p=((u+w),(v+x))
$$

Dengan menggunakan definisi $C_{j}$-pair teorema 1 dibuktikan dengan cara sebagai berikut,

$$
\begin{aligned}
q+p= & (u, v)+(w, x) \\
= & \left(\left(q_{0}+\boldsymbol{j} q_{2}\right)+\boldsymbol{i}\left(q_{1}+\boldsymbol{j} q_{3}\right)\right)+ \\
& \left(\left(p_{0}+\boldsymbol{j} p_{2}\right)+\boldsymbol{i}\left(p_{1}+\boldsymbol{j} p_{3}\right)\right) \\
= & \left(\left(q_{0}+\boldsymbol{j} q_{2}\right)+\left(p_{0}+\boldsymbol{j} p_{2}\right)\right)+ \\
& \boldsymbol{i}\left(\left(q_{1}+\boldsymbol{j} q_{3}\right)+\left(p_{1}+\boldsymbol{j} p_{3}\right)\right) \\
= & ((u+w),(v+x))
\end{aligned}
$$

\section{Pengurangan}

\section{Teorema 2}

Misalkan $q=(u, v), p=(w, x) \in \mathbb{C}_{j}$ dengan $u=q_{0}+\boldsymbol{j} q_{2}, v=q_{1}+\boldsymbol{j} q_{3}, w=p_{0}+\boldsymbol{j} p_{2}, x=$ $p_{1}+\boldsymbol{j} p_{3}$.

Maka

$$
q-p=((u-w),(v-x))
$$

\section{Bukti}

$$
\begin{aligned}
& q-p=(u, v)-(w, x) \\
& =\left(\left(q_{0}+\boldsymbol{j} q_{2}\right)+\boldsymbol{i}\left(q_{1}+\boldsymbol{j} q_{3}\right)\right)- \\
& \left(\left(p_{0}+\boldsymbol{j} p_{2}\right)+\boldsymbol{i}\left(p_{1}+\boldsymbol{j} p_{3}\right)\right)+ \\
& =\left(\left(q_{0}+\boldsymbol{j} q_{2}\right)-\left(p_{0}+\boldsymbol{j} p_{2}\right)\right)+ \\
& \boldsymbol{i}\left(\left(q_{1}+\boldsymbol{j} q_{3}\right)-\left(p_{1}+\boldsymbol{j} p_{3}\right)\right) \\
& =((u-w),(v-x))
\end{aligned}
$$

Pembuktian teorema 2 dibuktikan dengan menggunakan definisi notasi $C_{\boldsymbol{j}}-$ Pair dan sifat assosiatif bilangan quaternion.

\section{Konjugasi}

\section{Teorema 3}

Misalkan $q=(u, v), p=(w, x) \in \mathbb{C}_{j}$ dengan $u=q_{0}+\boldsymbol{j} q_{2}, v=q_{1}+\boldsymbol{j} q_{3}$ maka

\section{Bukti.}

$$
\begin{aligned}
& \bar{q}=q_{0}-\boldsymbol{i} q_{1}-\boldsymbol{j} q_{2}-\boldsymbol{k} q_{3} \\
& =q_{0}-\boldsymbol{j} q_{2}+\left(-\boldsymbol{i} q_{1}-\boldsymbol{k} q_{3}\right)
\end{aligned}
$$

$$
\begin{aligned}
& =q_{0}-\boldsymbol{j} q_{2}+\left(-\left(\boldsymbol{i} q_{1}+\boldsymbol{i} \boldsymbol{j} q_{3}\right)\right. \\
& =q_{0}-\boldsymbol{j} q_{2}+\left(-\boldsymbol{i}\left(q_{1}+\boldsymbol{j} q_{3}\right)\right. \\
& =\left(q_{0}-\boldsymbol{j} q_{2}\right)-\left(\boldsymbol{i}\left(q_{1}+\boldsymbol{j} q_{3}\right)\right. \\
& =\left(u^{\star},-v\right) .
\end{aligned}
$$

Dimana * adalah konjugasi klasik bilangan kompleks.

\section{Perkalian}

Misalkan $q=\left(u_{1}, v_{1}\right), \quad p=\left(u_{2}, v_{2}\right) \in \mathbb{C}_{j}$ dengan $u_{1}=q_{0}+\boldsymbol{j} q_{2}, \quad v_{1}=q_{1}+\boldsymbol{j} q_{3}, \quad u_{2}=$ $p_{0}+\boldsymbol{j} p_{2}, v_{2}=p_{1}+\boldsymbol{j} p_{3}$. Maka

$$
q p=\left(u_{1} u_{2}-v_{1}^{\star} v_{2}, u_{1}^{\star} v_{2}+v_{1} u_{2}\right)
$$

Bukti.

$$
\begin{aligned}
q p & \left(u_{1}, v_{1}\right)\left(u_{2}, v_{2}\right) \\
= & \left(\left(q_{0}+\boldsymbol{j} q_{2}\right)+\boldsymbol{i}\left(q_{1}+\boldsymbol{j} q_{3}\right)\right) \\
& \left(\left(p_{0}+\boldsymbol{j} p_{2}\right)+\boldsymbol{i}\left(p_{1}+\boldsymbol{j} p_{3}\right)\right) \\
= & \left(q_{0}+\boldsymbol{j} q_{2}\right)\left(\left(p_{0}+\boldsymbol{j} p_{2}\right)+\boldsymbol{i}\left(p_{1}+\boldsymbol{j} p_{3}\right)\right) \\
& +\boldsymbol{i}\left(q_{1}+\boldsymbol{j} q_{3}\right)\left(\left(p_{0}+\boldsymbol{j} p_{2}\right)+\boldsymbol{i}\left(p_{1}+\boldsymbol{j} p_{3}\right)\right) \\
= & \left(q_{0}+\boldsymbol{j} q_{2}\right)\left(p_{0}+\boldsymbol{j} p_{2}\right) \\
& +\left(q_{0}+\boldsymbol{j} q_{2}\right) \boldsymbol{i}\left(p_{1}+\boldsymbol{j} p_{3}\right) \\
& +\boldsymbol{i}\left(q_{1}+\boldsymbol{j} q_{3}\right)\left(p_{0}+\boldsymbol{j} p_{2}\right) \\
& +\boldsymbol{i}\left(q_{1}+\boldsymbol{j} q_{3}\right) \boldsymbol{i}\left(p_{1}+\boldsymbol{j} p_{3}\right) \\
= & \left(q_{0}+\boldsymbol{j} q_{2}\right)\left(p_{0}+\boldsymbol{j} p_{2}\right) \\
& +\boldsymbol{i}\left(q_{0}-\boldsymbol{j} q_{2}\right)\left(p_{1}+\boldsymbol{j} p_{3}\right) \\
& +\boldsymbol{i}\left(q_{1}+\boldsymbol{j} q_{3}\right)\left(p_{0}+\boldsymbol{j} p_{2}\right) \\
& +\boldsymbol{i} \boldsymbol{i}\left(q_{1}-\boldsymbol{j} q_{3}\right)\left(p_{1}+\boldsymbol{j} p_{3}\right) \\
= & \left(q_{0}+\boldsymbol{j} q_{2}\right)\left(p_{0}+\boldsymbol{j} p_{2}\right) \\
& -\left(q_{1}-\boldsymbol{j} q_{3}\right)\left(p_{1}+\boldsymbol{j} p_{3}\right) \\
& +\boldsymbol{i}\left(q_{0}-\boldsymbol{j} q_{2}\right)\left(p_{1}+\boldsymbol{j} p_{3}\right) \\
& +\boldsymbol{i}\left(q_{1}+\boldsymbol{j} q_{3}\right)\left(p_{0}+\boldsymbol{j} p_{2}\right) \\
= & \left\{\left(q_{0}+\boldsymbol{j} q_{2}\right)\left(p_{0}+\boldsymbol{j} p_{2}\right)\right. \\
& \left.-\left(q_{1}-\boldsymbol{j} q_{3}\right)\left(p_{1}+\boldsymbol{j} p_{3}\right)\right\} \\
& +\boldsymbol{i}\left\{\left(q_{0}-\boldsymbol{j} q_{2}\right)\left(p_{1}+\boldsymbol{j} p_{3}\right)\right. \\
& \left.+\boldsymbol{i}\left(q_{1}+\boldsymbol{j} q_{3}\right)\left(p_{0}+\boldsymbol{j} p_{2}\right)\right\} \\
= & \left(u_{1} u_{2}-v_{1}^{\star} v_{2}, u_{1}^{\star} v_{2}+v_{1} u_{2}\right) \\
& +
\end{aligned}
$$

\section{Perkalian Bikompleks Quaternion}

Seperti dijelaskan sebelumnya, bahwa perkalian bikompleks quaternion merupakan kasus khusus dalam perkalian quaternion, hal ini dikarenakan pada kasus perkalian bikompleks quaternion berlaku sifat komutatif. Untuk menyatakan perkalian bikompleks quaternion, digunakan simbol “ $\bigotimes$ ”. Sebelum melakukan perkalian bikompleks quaternion, maka terlebih dahulu, didefinisikan beberapa aturan perkalian berikut, $\boldsymbol{i} \otimes \boldsymbol{j}=\boldsymbol{j} \otimes \boldsymbol{i}=\boldsymbol{k}$, 
$\boldsymbol{j} \otimes \boldsymbol{k}=\boldsymbol{k} \otimes \boldsymbol{j}=\boldsymbol{i}$,

$\boldsymbol{i} \otimes \boldsymbol{k}=\boldsymbol{k} \otimes \boldsymbol{i}=-\boldsymbol{j}$,

$i \otimes i=j \otimes j=-1$,

$\boldsymbol{k} \otimes \boldsymbol{k}=\mathbf{1}$.

Selanjutnya, akan digunakan sifat-sifat tersebut untuk membuktikan beberapa teorema berikut.

\section{Teorema 4.}

Misalkan $\quad q=\left(u_{1}, v_{1}\right), \quad p=\left(u_{2}, v_{2}\right) \in \mathbb{C}_{j}$ dengan $u_{1}=q_{0}+\boldsymbol{j} q_{2}, \quad v_{1}=q_{1}+\boldsymbol{j} q_{3}, \quad u_{2}=$ $p_{0}+\boldsymbol{j} p_{2}, v_{2}=p_{1}+\boldsymbol{j} p_{3}$. Maka

$$
p \otimes q=\left(u_{1} u_{2}-v_{1} v_{2}, u_{1} v_{2}+v_{1} u_{2}\right)
$$

\section{Bukti.}

$$
\begin{aligned}
& q \otimes p=\left(u_{1}, v_{1}\right)\left(u_{2}, v_{2}\right) \\
&=\left(\left(q_{0}+\boldsymbol{j} q_{2}\right)+\boldsymbol{i}\left(q_{1}+\boldsymbol{j} q_{3}\right)\right) \\
&\left(\left(p_{0}+\boldsymbol{j} p_{2}\right)+\boldsymbol{i}\left(p_{1}+\boldsymbol{j} p_{3}\right)\right) \\
&=\left(q_{0}+\boldsymbol{j} q_{2}\right)\left(\left(p_{0}+\boldsymbol{j} p_{2}\right)+\boldsymbol{i}\left(p_{1}+\boldsymbol{j} p_{3}\right)\right) \\
&+\boldsymbol{i}\left(q_{1}+\boldsymbol{j} q_{3}\right)\left(\left(p_{0}+\boldsymbol{j} p_{2}\right)+\boldsymbol{i}\left(p_{1}+\boldsymbol{j} p_{3}\right)\right) \\
&=\left(q_{0}+\boldsymbol{j} q_{2}\right)\left(p_{0}+\boldsymbol{j} p_{2}\right) \\
&+\left(q_{0}+\boldsymbol{j} q_{2}\right) \boldsymbol{i}\left(p_{1}+\boldsymbol{j} p_{3}\right) \\
&+\boldsymbol{i}\left(q_{1}+\boldsymbol{j} q_{3}\right)\left(p_{0}+\boldsymbol{j} p_{2}\right) \\
&+\boldsymbol{i}\left(q_{1}+\boldsymbol{j} q_{3}\right) \boldsymbol{i}\left(p_{1}+\boldsymbol{j} p_{3}\right) \\
&=\left(q_{0}+\boldsymbol{j} q_{2}\right)\left(p_{0}+\boldsymbol{j} p_{2}\right) \\
&+\boldsymbol{i}\left(q_{0}+\boldsymbol{j} q_{2}\right)\left(p_{1}+\boldsymbol{j} p_{3}\right) \\
&+\boldsymbol{i}\left(q_{1}+\boldsymbol{j} q_{3}\right)\left(p_{0}+\boldsymbol{j} p_{2}\right) \\
&+\boldsymbol{i} \boldsymbol{i}\left(q_{1}+\boldsymbol{j} q_{3}\right)\left(p_{1}+\boldsymbol{j} p_{3}\right) \\
&=\left(q_{0}+\boldsymbol{j} q_{2}\right)\left(p_{0}+\boldsymbol{j} p_{2}\right) \\
&-\left(q_{1}+\boldsymbol{j} q_{3}\right)\left(p_{1}+\boldsymbol{j} p_{3}\right) \\
&+\boldsymbol{i}\left(q_{0}+\boldsymbol{j} q_{2}\right)\left(p_{1}+\boldsymbol{j} p_{3}\right) \\
&+\boldsymbol{i}\left(q_{1}+\boldsymbol{j} q_{3}\right)\left(p_{0}+\boldsymbol{j} p_{2}\right) \\
&=\left(\left(q_{0}+\boldsymbol{j} q_{2}\right)\left(p_{0}+\boldsymbol{j} p_{2}\right)\right. \\
&\left.-\left(q_{1}+\boldsymbol{j} q_{3}\right)\left(p_{1}+\boldsymbol{j} p_{3}\right)\right) \\
&+\boldsymbol{i}\left(\left(q_{0}+\boldsymbol{j} q_{2}\right)\left(p_{1}+\boldsymbol{j} p_{3}\right)\right. \\
&\left.+\left(q_{1}+\boldsymbol{j} q_{3}\right)\left(p_{0}+\boldsymbol{j} p_{2}\right)\right) \\
&=\left(u_{1} u_{2}-v_{1} v_{2}, u_{1} v_{2}+v_{1} u_{2}\right) \\
&+\left(v_{1} a w a\right. \\
&
\end{aligned}
$$

Langkah awal pembuktian dilakukan dengan menggunakan definisi $C_{j}-$ Pair. Selanjutnya, langkah 2, 3, 4, 5 dan 6 digunakan sifat komutatif, asosiatif dan distributf perkalian.

Dengan cara yang sama, akan dengan mudah diperlihatkan bahwa, $q \otimes p=p \otimes q$.

\section{Teorema 5.}

Misalkan, diberikan $q=\left(q_{1}, q_{2}\right)=(a+$ $\boldsymbol{j} c, b+\boldsymbol{j} d)$, untuk sebarang $\mathbb{C}_{\boldsymbol{i}}$ bilangan $z=$ $\mathcal{R}(z)+\boldsymbol{i} \mathcal{J}_{\boldsymbol{i}}(z)=\left(\mathcal{R}(z), \mathcal{J}_{\boldsymbol{i}}(z)\right)$, maka $q \otimes z$

$$
=\left(q_{1} \mathcal{R}(z)-q_{2} \mathcal{J}_{\boldsymbol{i}}(z),\left(q_{1} \mathcal{J}_{\boldsymbol{i}}(z)+q_{2} \mathcal{R}(z)\right)\right)
$$

Bukti.

$q \otimes z=(a+\boldsymbol{j} c, b+\boldsymbol{j} d) \otimes\left(\mathcal{R}(z), \mathcal{J}_{\boldsymbol{i}}(z)\right)$, $=((a+j c)+\boldsymbol{i}(b+\boldsymbol{j} d))\left(\mathcal{R}(z)+\boldsymbol{i} \jmath_{\boldsymbol{i}}(z)\right)$

$=(a+\boldsymbol{j} c) \mathcal{R}(z)+(a+\boldsymbol{j} c) \boldsymbol{i} \boldsymbol{\jmath}_{\boldsymbol{i}}(z)$

$+\boldsymbol{i}(b+\boldsymbol{j} d) \mathcal{R}(z)+\boldsymbol{i}(b+\boldsymbol{j} d) \boldsymbol{i}_{\boldsymbol{i}}(z)$

$=(a+\boldsymbol{j} c) \mathcal{R}(z)-(b+\boldsymbol{j} d) \mathcal{J}_{\boldsymbol{i}}(z)$

$+(a+\boldsymbol{j} c) \boldsymbol{i} \mathcal{J}_{\boldsymbol{i}}(z)+\boldsymbol{i}(b+\boldsymbol{j} d) \mathcal{R}(z)$

$=(a+\boldsymbol{j} c) \mathcal{R}(z)-(b+\boldsymbol{j} d) \mathcal{J}_{\boldsymbol{i}}(z)$

$+\boldsymbol{i}\left((a+\boldsymbol{j} c) \mathcal{J}_{\boldsymbol{i}}(z)+(b+\boldsymbol{j} d) \mathcal{R}(z)\right)$

$=q_{1} \mathcal{R}(z)-q_{2} \mathcal{J}_{\boldsymbol{i}}(z)$

$+\boldsymbol{i}\left(q_{1} \mathcal{J}_{\boldsymbol{i}}(z)+q_{2} \mathcal{R}(z)\right)$

$=\left(q_{1} \mathcal{R}(z)-q_{2} \mathcal{J}_{\boldsymbol{i}}(z),\left(q_{1} \mathcal{J}_{\boldsymbol{i}}(z)+q_{2} \mathcal{R}(z)\right)\right)$ Teorema 5 , dibuktikan dengan menggunakan definisi $\quad C_{\boldsymbol{j}}$-Pair, karena $\boldsymbol{i} \otimes \boldsymbol{i}=-1$ dihasilkan langkah ke-3. Selanjutnya, langkah ke-3, 4, 5 digunakan sifat asosiatif dan komutatif perkalian.

\section{Teorema 6.}

Misalkan, diberikan $q=\left(q_{1}, q_{2}\right)=(a+$ $\boldsymbol{j} c, b+\boldsymbol{j} d)$, untuk sebarang $\mathbb{C}_{\boldsymbol{j}}$ bilangan $w=$ $\mathcal{R}(w)+\boldsymbol{j}_{\boldsymbol{i}}(w)=(w, 0)$, maka

$$
q \otimes w=\left(q_{1} w, q_{2} w\right)
$$

\section{Bukti.}

Teorema 6 dibuktikan dengan cara sebagai berikut,

$q \otimes w=\left(q_{1}, q_{2}\right) \otimes(w, 0)$

$=(a+\boldsymbol{j} c, b+\boldsymbol{j} d)(w, 0)$

$=(((a+j c) w+(a+j c) 0)$,

$(b+\boldsymbol{j} d) w+(b+\boldsymbol{j} d) 0)$

$=(a+\boldsymbol{j} c) w,(b+j d) w$

$=\left(q_{1} w, q_{2} w\right)$

\section{Teorema 7.}

Misalkan, diberikan $q=\left(q_{1}, q_{2}\right)=(a+$ $\boldsymbol{j} c, b+\boldsymbol{j} d)$, untuk sebarang $\mathbb{C}_{\boldsymbol{k}}$ bilangan $s=$ $\mathcal{R}(s)+\boldsymbol{k} \mathcal{J}_{\boldsymbol{k}}(s)=\left(\mathcal{R}(s), \boldsymbol{j} \mathfrak{J}_{\boldsymbol{k}}(s)\right)$, maka

$q \otimes s=\left(q_{1}\right) \mathcal{R}(s)-\boldsymbol{j} q_{2} \mathcal{J}_{\boldsymbol{k}}(s)$,

$\boldsymbol{i}\left(q_{2} \mathcal{R}(s)+\boldsymbol{j} q_{1} \mathcal{J}_{\boldsymbol{k}}(s)\right)$

\section{Bukti.}

$q \otimes s=((a+\boldsymbol{j} c),(b+\boldsymbol{j} d))\left(\mathcal{R}(s), \boldsymbol{j} \mathcal{J}_{\boldsymbol{k}}(s)\right)$ $={ }^{1}((a+\boldsymbol{j} c)+\boldsymbol{i}(b+\boldsymbol{j} d))\left(\mathcal{R}(s)+\boldsymbol{k J}_{\boldsymbol{k}}(s)\right)$ 


$$
\begin{aligned}
& ={ }^{2}((a+\boldsymbol{j} c))\left(\mathcal{R}(s)+\boldsymbol{k J}_{\boldsymbol{k}}(s)\right) \\
& +(\boldsymbol{i}(b+\boldsymbol{j} d))\left(\mathcal{R}(s)+\boldsymbol{k}_{\boldsymbol{k}}(s)\right) \\
& ={ }^{3}\left((a+\boldsymbol{j} c) \mathcal{R}(s)+\boldsymbol{i} \boldsymbol{j}(a+\boldsymbol{j} c) \mathcal{J}_{\boldsymbol{k}}(s)\right) \\
& +\boldsymbol{i}(b+\boldsymbol{j} d) \mathcal{R}(s)-\boldsymbol{j}(b+\boldsymbol{j} d) \mathcal{J}_{\boldsymbol{k}}(s) \\
& ={ }^{4}\left((a+\boldsymbol{j} c) \mathcal{R}(s)-\boldsymbol{j}(b+\boldsymbol{j} d) \mathcal{J}_{\boldsymbol{k}}(s)\right) \\
& +\boldsymbol{i}\left((b+\boldsymbol{j} d) \mathcal{R}(s)+\boldsymbol{j}(a+\boldsymbol{j} c) \mathcal{J}_{\boldsymbol{k}}(s)\right) \\
& ={ }^{5}\left(\left(q_{1}\right) \mathcal{R}(s)-\boldsymbol{j} q_{2} \mathcal{J}_{\boldsymbol{k}}(s)\right) \\
& +\boldsymbol{i}\left(q_{2} \mathcal{R}(s)+\boldsymbol{j} q_{1} \mathcal{J}_{\boldsymbol{k}}(s)\right) \\
& ={ }^{6}\left(q_{1}\right) \mathcal{R}(s)-\boldsymbol{j} q_{2} \mathcal{J}_{\boldsymbol{k}}(s), \\
& \boldsymbol{i}\left(q_{2} \mathcal{R}(s)+\boldsymbol{j} q_{1} \mathcal{J}_{\boldsymbol{k}}(s)\right)
\end{aligned}
$$

Langkah 1 digunakan definisi notasi $C_{j}-$ Pair, sedangkan langkah 2, 3, 4, 5 dan 6, digunakan sifat komutatif, asosiatif dan distributf quaternion dengan memperhatikan operasi bikompleks quaternion.

\section{Teorema 8.}

Misalkan, diberikan $q=\left(q_{1}, q_{2}\right)=(a+$ $\boldsymbol{j} c, b+\boldsymbol{j} d)$, dan sebaran bilangan $\boldsymbol{k}=(0, \boldsymbol{j})$, maka $q \otimes \boldsymbol{k}$ dinyatakan dengan,

$$
q \otimes \boldsymbol{i}=\left(-q_{2}, q_{1}\right)
$$

\section{Bukti.}

$$
\begin{aligned}
& q \otimes \boldsymbol{i}=\left(q_{1}, q_{2}\right) \otimes(0, \boldsymbol{j}) \\
& =((a+\boldsymbol{j} c)+\boldsymbol{i}(b+\boldsymbol{j} d))(0+\boldsymbol{i}) \\
& =(\boldsymbol{i}(a+\boldsymbol{j} c)-(b+\boldsymbol{j} d)) \\
& =(-(b+\boldsymbol{j} d)+\boldsymbol{i}(a+\boldsymbol{j} c)) \\
& =\left(-q_{2}, q_{1}\right)
\end{aligned}
$$

\section{Teorema 9.}

Misalkan, diberikan $q=\left(q_{1}, q_{2}\right)=(a+$ $\boldsymbol{j} c, b+\boldsymbol{j} d)$, dan sebaran bilangan $\boldsymbol{j}=(\boldsymbol{j}, 0)$, maka $q \otimes \boldsymbol{j}$ dinyatakan dengan

$$
q \otimes \boldsymbol{j}=\left(q_{1} \boldsymbol{j}, \boldsymbol{j} q_{2}\right)
$$

\section{Bukti.}

$$
\begin{aligned}
q \otimes \boldsymbol{j} & =\left(q_{1}, q_{2}\right) \otimes(\boldsymbol{j}, 0) \\
& =((a+\boldsymbol{j} c)+\boldsymbol{i}(b+\boldsymbol{j} d))(\boldsymbol{j}+\boldsymbol{i} 0) \\
& =(a+\boldsymbol{j} c) \boldsymbol{j}+\boldsymbol{i} \boldsymbol{j}(b+\boldsymbol{j} d) \\
& =q_{1} \boldsymbol{j}+\boldsymbol{i} \boldsymbol{j} q_{2} \\
& =\left(q_{1} \boldsymbol{j}, \boldsymbol{j} q_{2}\right)
\end{aligned}
$$

\section{Teorema 10.}

Misalkan, diberikan $q=\left(q_{1}, q_{2}\right)=(a+$ $\boldsymbol{j} c, b+\boldsymbol{j} d)$, dan sebaran bilangan $\boldsymbol{j}=(\boldsymbol{j}, 0)$, maka $q \otimes \boldsymbol{j}$ dinyatakan dengan

$$
q \otimes \boldsymbol{j}=\left(-\boldsymbol{j} q_{2}, \boldsymbol{j} q_{1}\right)
$$

\section{Bukti.}

$$
\begin{aligned}
q \otimes \boldsymbol{j} & =\left(q_{1}, q_{2}\right) \otimes(\boldsymbol{j}, 0) \\
& =((a+\boldsymbol{j} c)+\boldsymbol{i}(b+\boldsymbol{j} d))(0+\boldsymbol{i} \boldsymbol{j}) \\
& =(a+\boldsymbol{j} c) \boldsymbol{i} \boldsymbol{j}+\boldsymbol{i} \boldsymbol{i} \boldsymbol{j}(b+\boldsymbol{j} d) \\
& =\boldsymbol{i}(a+\boldsymbol{j} c) \boldsymbol{j}-\boldsymbol{j}(b+\boldsymbol{j} d) \\
& =-\boldsymbol{j}(b+\boldsymbol{j} d)+\boldsymbol{i} \boldsymbol{j}(a+\boldsymbol{j} c) \\
& =\left(-\boldsymbol{j} q_{2}, \boldsymbol{j} q_{1}\right)
\end{aligned}
$$

\section{KESIMPULAN}

Berdasarkan pembahasan pada bagian sebelumnya disimpulkan bahwa, notasi $C_{\boldsymbol{j}}-$ pair merupakan salah satu rujukan yang bisa digunakan untuk merepresentasikan bilangan quaternion. Pada bagian akhir pembahsan dilakukan notasi $C_{j}$-pair pada perkalian bikompleks quaternion yang dimana merupakan kasus khusus dari perkalian quaternion.

\section{DAFTAR PUSTAKA}

[1] Bahri, M,. Ashino, R., and Vailancourt, R. 2013. Convolution theorems for quaternion Fourier transform, properties and applications, Hindawi Publishing Corporation Abstact and Applied Analysis, vol. 2013, ID 162769 : 1-2.

[2] Bahri, M., Ashino, R. 2013. Correlation theorems for type II quaternion Fourier transform, International conference on wavelet analysis and pattern recognition. 136-141.

[3] Bahri, M., Hitzer, Ashino, R., and Hayasi, A. 2008. An uncertainty principle for quaternion fourier transform. Computer and Mathematics with Applications. 56: 2398.

[4] Hitzer, E. M. S. 2013. Quaternion Fourier Trasform on Quaternion Field and Generalization. Advance Applications of Clifford Algebras. 17: 120.

[5] Muh. Irwan. dan Muhammad Ridwan. 2017. Transformasi Fourier dan Transformasi Fourier Quaternion, Jurnal Matematika Statistika dan Analisis Vol 5 No 2 (2017). 
JURNAL MSA VOL. 6 NO. 1 ED. JAN-JUNI 2018

[6] Muh. Irwan. 2015. Quaternion and it's properties, Jurnal Matematika Statistika dan Analisis Vol 3 No 1 (2017).

[7] Morais, J.P., Geogiev S., Sprosieg, W. 2012. Real Quaternionic Calculus Handbook. Springer Basel: Hidelberg New York London.

[8] Todd, dkk. 2014. Quaternion Fourier Transform for Signal and Image Processing, Fokus 